\title{
How to be Wise without Being a Wise-Guy: Developing the The 'Infi-Net'
}

\section{Ronald Glasberg}

\author{
Associate Professor \\ Department of Communication, Media and Film \\ The University of Calgary \\ 2500 University Drive NW \\ Calgary, Alberta T2N $3 L 1$ \\ Canada
}

\section{Doi: 10.36941/ajis-2019-0001}

\begin{abstract}
The goal of the paper is to develop a metaphilsophical approach to wisdom by formulating a conceptual array (intra-personal, inter-personal, intra-cultural and inter-cultural) designed to apprehend an infinite whole - i.e., an 'infi-net'. The key is to balance depth and breadth by having the conceptual array interconnected with respect to itself at the same time that it is open to isomorphically similar sets that are also inter-connected. Wisdom here is a systemic way of breaking out of narrow and rigid ways of thinking by learning to use concepts that are broad in range but also sensitive to the differences that divide us. If the wise-guy is a cynic because the possibilities of an integrative overview are dismissed out of hand, the truly wise person is a creator of holistic perspectives that serve to facilitate communication.
\end{abstract}

Keywords: interdisciplinarity, unity of knowledge, wisdom, holistic thinking, disciplinary theory

\section{Introduction}

To catch a small fish, a net of small weave is in order; and while that might serve to trap larger fish, too fine a weave might render the net overly feeble with respect to stronger quarry. Thus, to catch a bigger fish a larger and stronger net is required. To capture the greatest of fish, the greatest of nets must be designed and constructed albeit in a manner that does not allow the smaller fish to slither through.

This discussion is not about catching fish. It is about systematically gaining the insight associated with wisdom, which I am here defining as the broadest and most profound understanding of the whole. Broadness usually comes with a sacrifice of depth (or profundity) and vice versa. Wisdom must entail harnessing both of these aspects of knowing and learning how to manage this tensive team in such a manner as to pull forward one's mind - one's intellectual chariot so to speak - in the direction of truth.

If the whole is without limit, the net must be of a weave that is appropriate - an 'infi-net' (to coin a term that plays with the concepts of 'infinitude' and 'net'). The present discussion aims to offer such a net of concepts - a net designed to catch in a tentative fashion the infinitely large fish of total or absolute knowledge without letting too many of the smaller ones escape. It is necessarily an ongoing project. For the infi-net is constantly renewed every time that it is cast into the sea of truth and drawn back into the ship of consciousness to see what has been caught. Tares in the fabric are mended while the warp and woof are re-woven before the next recasting. The process or repair and redesign is endless. But then what would one expect of an infi-net? 
To help the reader with what is to come, I am going to present the net early on in this discussion rather than at the end. For it seems to me that a piece-meal presentation would be overly taxing because there are so many challenging concepts (some of my own devising) to hold in one's mind at the same time. One must get a sense of how these categories of analysis hang together as a whole before one can begin to situate the parts in a more 'user-friendly' manner.

The infi-net is composed of broad-ranging categories, but these categories are themselves placed in a framework of 'meta-categories' that constitute the warp and woof of the net's conceptual structure. After presenting a grid that illustrates how categories and meta-categories are connected to each other, I will provide a set of definitions for all of these perhaps unusual terms. This will be followed by more detailed elaborations and examples that show how the net actually functions in its integrative capacity.

\begin{tabular}{|l|l|l|l|l|}
\hline Meta-Categories & $\begin{array}{l}\text { (A) Emergent } \\
\text { Externality }\end{array}$ & $\begin{array}{l}\text { (B) Emergent } \\
\text { Internality }\end{array}$ & $\begin{array}{l}\text { (C) Foundational } \\
\text { Externality }\end{array}$ & $\begin{array}{l}\text { (D) Foundational } \\
\text { Internality }\end{array}$ \\
\hline $\begin{array}{l}\text { (1) Self-Society } \\
\text { Dimensional Hierarchy }\end{array}$ & $\begin{array}{l}\text { A1 Intra- } \\
\text { personal }\end{array}$ & B1 Inter-personal & C1 Intra-cultural & $\begin{array}{l}\text { D1 Inter- or Extra- } \\
\text { cultural }\end{array}$ \\
\hline $\begin{array}{l}\text { (2) Fundamental Cultural } \\
\text { Assumptions }\end{array}$ & A2 Life-death & $\begin{array}{l}\text { B2 Freedom- } \\
\text { slavery }\end{array}$ & C2 Wisdom-folly & D2 Good-evil \\
\hline $\begin{array}{l}\text { (3) Disciplinary Orientation } \\
\text { (4) Primary Relation to }\end{array}$ & A3 Science & B3 Art & C3 Philosophy & D3 Religion \\
\hline
\end{tabular}

The foregoing grid represents the infi-net, where $A, B, C, D$ as well as $1,2,3,4$ are associated with the meta-categories that are designed to enclose a set of more minor categories: A1, A2, etc. B1, B2, etc., C1, C2, etc., and D1, D2, etc. in the format of a matrix.

After defining the vertical set of meta-categories $(1,2,3$, and 4$)$, I will then turn to the horizontal set $(A, B, C$, and $D)$. The discussion of the more basic categories will follow.

\section{Vertical Meta-Categories}

Definition \#1 - Self-Society Dimensional Hierarchy: By this term I mean a 'self-society' set of relationships that organizes concepts in a manner that moves from the intra-personal to the interpersonal and then to the intra-cultural and concluding with the inter- or extra-cultural. While the foregoing subsidiary categories will be defined at the appropriate time, the meta-categorical framework is clearly hierarchical and dimensional in nature: hierarchical in the sense that the 'intrapersonal' is lower in the hierarchy than the more inclusive network of inter-personal relationships, etc.; dimensional in the sense that the concepts at the higher levels include those in the lower levels (e.g., as the second dimension of a plane includes or encompasses the first dimension of many lines). To elaborate on the hierarchical aspect, categories at the apex are those that sum up the values of a civilization as a whole and thus have a certain power or authority over those that are focused on how a particular individual makes sense of his or her life.

Definition \#2 - Fundamental Cultural Assumptions: By this I mean the set of presuppositions that underlie (consciously or unconsciously) the main concerns of life in a given cultural configuration. These concerns (life-death, freedom-slavery, wisdom-folly, and good-evil) will be defined at the appropriate time and constitute the basic sub-categories existing within the framework of this meta-category.

Definition \#3 - Disciplinary Orientation: By this term I mean the primary forms of knowing and/or understanding the world as these forms are situated in disciplines that are significantly different from each other and together provide a comprehensive vision or the whole. Again the key elements (science, art, philosophy, and religion) will be defined at the appropriate time.

Definition \#4 - Primary Relation to Reality: By this I mean fundamental psychological relationships to reality that reflect our deepest aspirations or goals: mastery, intimacy, transcendence, and surrender.

It is important to note that the vertical set of meta-categories is not meant to be exhaustive. 
Although other sets could be added, these must be structured so as to essentially be quaternal in nature. That is, any given vertical set must accommodate the four-fold structure of the horizontal set. Otherwise the integral structure of the infi-net, which is matrix-like, would become incoherent.

\section{Horizontal Meta-Categories}

Definition \#5 - Externality-Internality: although connected to 'emergence-foundation' counterparts in the top line of the grid, this pair needs to be defined separately. While the vertical sets of metacategories are relatively independent with respect to each other, the horizontal set is characterized by a kind of complementary inter-dependence. To bring out this inter-dependence a more extensive definition is required, and the following points thus function as a kind of explication rather than a simple definition that would mislead by way of its truncation.

a) Internality and externality can no more be defined apart from each than can yin and yang, which may be said to resonate with the foregoing pair in an isomorphic fashion insofar as yin is a kind of hollow, receptive, or internal space, which may be penetrated or filled up by a yang force that is external to it.

b) Thus the internal is a kind of shared or common space, and the external is that which is outside that space. For example, sharing a common language is a manifestation of internality with respect to those who are outside of that linguistic community and hence external to it. Likewise, being with others inside a physical room is a kind of internality with respect to those who are situated outside of it (i.e., external to it).

c) While internality has significant manifestations in the physical world, it is more pertinent to the sphere of the mind or consciousness insofar as our individual selves are an integral collection of thoughts, feelings, memories, etc. that appear to occupy a common space from which other selves and the physical world are excluded unless protocols of connection are established.

d) If I do not use mind or consciousness in place of internality, it is because mind or consciousness do not automatically entail the complementary counterpart of externality. In other words, whether the internal is mental or material in nature, one cannot think of internal without asking, 'internal' with respect to what 'external' and vice versa. No doubt, some categorizers can weave their nets in such a manner that externality is taken to be universal and internality is a taken to be a delusion (and, of course, vice versa); but if wisdom entails grasping the widest range of phenomena and integrating these in a coherent manner, the infi-net will be constructed with a view to inclusivity and thus avoid reducing internality to externality (or vice versa).

e) The 'realm' of the internal (if I might use a non-spatial term such as 'realm') also has qualities that distinguish it from its external counterpart. In the latter, objects are usually separable from each other and have boundaries that are more or less stable and/or well defined. In the former, this is not always or even usually the case. For example, the 'thought' of an apple in the internal world is not like a real physical apple in the external world. The physical apple is separate from other objects and offers a certain resistance to our will. This is because thoughts, although they can be differentiated from each other, have no boundaries. As a result, the creative power that is characteristic of the internal can play with its object by altering it at will in a manner that is not possible in the external realm. If I were to give this quality a name, it would be 'indeterminate boundary phenomena'. Thus, the ability to define something in a more or less unambiguous way pertains more to the external world; and when this 'definitional' capacity is directed toward the internal (from which it emerged), it is at a certain disadvantage because commonspace phenomena in the internal realm and, indeed, the internal realm itself become unavoidably distorted in the wake of definitions that attempt to look at the internal from the outside (i.e., externalistically).

f) The one exception to the rule of 'indeterminate boundary phenomena' - and it is a crucial exception - is of course thoughts pertaining to mathematics, where definitions are obviously rigorous and, indeed, more rigorous than would be the case with respect to 
phenomena in the external world. However, without venturing too deeply into an area of utter profundity beyond the scope of the present discussion, the internal as common space in mathematics asserts itself by way of the centrality of the 'equal' sign (i.e., '='). If entities can be equated, they manifest a quality of being in a common space in an absolute way because they are in essence shown to be one and the same by way of some kind of mathematical equivalence. In contrast to this, the inhabitants of the external realm show themselves, while separable from each other, to have quantitative aspects (length, mass, motion, etc.) that are conflated in common objects unless experimental protocols devise ways of isolating these qualities so that they may be subject to measurement. It is from such measurement that scientific laws are put forward. Inasmuch as these 'laws' bear the mark of the equal sign (i.e., '='), certain areas of the external world have been internalized via the common-space quality of mathematics.

g) As for 'awareness' being a key term for understanding the internal in its mind-like aspect, it is problematic to the extent that it distracts one from understanding the foregoing qualities of internality (i.e., its complementarity with externality, its indeterminate boundary resistance to definition, its capacity for absolute unity via mathematical unity). At the same time, if awareness is to play a significant role in grasping internality, it needs to be placed in the context of two important modifiers: that of the self and that of the non-self. With respect to the former, I am referring to the common space within the construct known as the self. Here exist personal memories, intimations of the future, feelings, sensations, thoughts, judgments, concepts, etc., but these elements that are internal with respect to the construct known as the self are not static. They are constantly evolving in accord with our life experiences, and the common space within which that evolution takes place is creatively coordinated to bring these elements into some kind of coherence or harmony. In other words, awareness in the context of the self is not an exercise in passivity. It is an ongoing act of creativity - creativity that also manifests itself in the act of dreaming where a whole world is created. (To illustrate the power of dream creativity, one only has to note that a shift in consciousness associated with waking up is required for the dreamer to realize that the world created in the dream was not real.) By the same token, the creation of mathematical abstractions characterized by common-space equivalencies bespeaks a comparable creative capacity that is at odds with the passivity associated with the unmodified term, awareness.

However, when shifting our focus from the self-aspect of internality as a place of creativity to the area of the non-self, awareness is best understood from a more Buddhist perspective where the creative dimension of internality is purposefully suppressed, not only with respect to the construct known as the self, but also with respect to the those constructs which are the occasion for creativity. What has happened, then, to the common-space aspect of internality? At the risk of gross overs-simplification, I would venture to suggest that the scope of internality has expanded to encompass a non-self and what might best be described as the totality of being. (Hagen, pp. 46, 162) In other words, common-space has become a oneness with being that is experienced as a blissful wakefulness or nirvana, where the creative aspect of internality has been replaced with what might be called a purely reflective state. My point here is that awareness, when coupled with the foregoing modifiers (i.e., self and non-self), is seen as more creatively active and spiritually expansive than as essentially passive in nature.

Summary of Definition \#5: If I appear to have spent an in-ordinate amount of time on the internal-external definition, it is not just because of an inherent subtlety that these inter-dependent terms exhibit. It is also because internality, as an integral aspect of reality, is being downplayed at the present time. The rise of Al (artificial intelligence) and the neurological approach to consciousness are not to be shunned because they have, as a kind of project, the reduction of consciousness (internality) to computer or brain phenomena (externality). However, just as I believe the internal can be explored with respect to the external, I also believe it is a mark of wisdom to balance this by exploring the external with respect to the internal. A wise-guy is one who takes on a kind of mocking attitude to what is deemed false; and to the extent that such an attitude is a function of one's historical and cultural situation, wisdom calls for a broader and more balanced 
perspective. In this case, I am seeking to present the internal, not as a trick of language or as some kind of illusion, but as a necessary correlate of the external - a correlate that has its own unique set of qualities that I have spent much time elaborating. The proof of their value, however, can only come from seeing how they function in the framework of the matrix being constructed.

Definition \#6 - Emergent-Foundational: although these meta-categories are connected to 'internal-external', they can also be understood in their own right. Just as reality has an interdependent complementary quality (i.e., internality-externality, discussed in Definition \#5), it also has a kind of procreative aspect, where foundations underlie and generate something that may be said to emerge from these foundations. Thus, the foundational pertains to what might be called a deep or 'primary' level of being while the emergent may be associated with what flows from that deeper level and is hence derivative or secondary. As one might expect, these terms have a strongly relativist aspect (as do internal and external). For what appears foundational from one perspective might be emergent from another. For example, atoms might be foundational with respect to everyday objects (e.g., tables and chairs) but emergent with respect to the protons, neutrons, and electrons that function as their building-blocks.

Integrating Definition \#5 with \#6 -- (A) Emergent-Exernality, (B) Emergent-Internality, (C) Foundational-Externality, (D) Foundational-Internality: As can be seen, these four meta-categories can function as a matrix in and of themselves. However, by placing them in the top row of the infinet matrix, I am testing their ability to organize an endless set of subsequent rows, four of which I have defined above (Definitions \#1 to \#4). If they succeed in shedding an integrative light on (1) the Self-Society Dimensional Hierarchy, (2) Fundamental Cultural Assumptions, (3) Disciplinary Orientation, and (4) Primary Relation to Reality, the value of the top row of the infi-net will be vindicated. But wisdom here is not a matter of reduction, where the elements of each row are turned into expressions of what lies at the top row. Wisdom, to me, is matter of enhanced communication wherein the function of the infi-net is to facilitate communication between all the elements strategically situated within the grid. With this in mind, let us begin the presentation.

\section{Exploring the Infi-net}

\subsection{The Self-Society Dimensional Hierarchy}

The first row of the infi-net that I will be coordinating with the horizontal meta-categories (Emergent Externality, Emergent Internality, etc.) is that associated with what I have termed the Self-Society Dimensional Hierarchy (Definition \#1). It is dimensional insofar as the self is a primary dimension of being that is contained by a secondary dimension of other selves who are interacting with each other. The third dimension is that of culture (i.e., the intra-cultural), which places the foregoing interactions in a larger framework, where the second or inter-personal level is guided by deeper principles or purposes that the culture articulates in a variety of ways. This third dimension is subsumed by yet a fourth, which I call inter- or trans-cultural because it is associated with cultural goals in a way that makes implicit or explicit reference to the principles animating other cultures or to a deeper level of reality that transcends culture altogether. The dimensional aspect that animates this set of categories is supplemented by a hierarchical quality in the sense that the higher dimensions encompass greater ranges of experience, with the fourth dimension being the most totalistic.

How then might these categories correlate with the horizontal set of meta-categories?

Turning first to the intra-personal category, we are in the world of the individual, but not with a view to exploring the common space within. In the dimensional context of this set of categories, the intra-personal bespeaks externality with respect to the second or inter-personal dimension. This is because individuality or personal identity only makes sense when seen as external to or not in a common space with others. That common space only comes into play when there is some kind of integral relationship with others and an internal sphere is accordingly brought into being in the context of various inter-personal relationships (e.g., family, neighbors, clubs, political parties, etc.). The intra-personal, if viewed in isolation and not as part of the infi-net, is more internal. But that is not how it is being viewed here. In a relativistic sense the intra-personal is more externalistic in 
nature insofar as all individuals have a quality of uniqueness or mutual externality with respect to each other - an externality that is mitigated by the common space that can be established at the inter-personal level. In other words, individuals and groups - the first two levels of the hierarchy emerge from or are derived from deeper realities associated with history, geography, language, etc. They are also emergent in the sense that they create themselves in the context of the immediate challenges that confront them with respect to the foregoing elements.

Thus, when it comes to the third and fourth dimensions of the self-society hierarchy, culture is foundational with respect to those who are born and raised within its ambit. But whereas any culture in and of itself is different from others, this foundational reality is essentially externalistic even if it does provide a kind of common space for those living within it. If I do not emphasize the latter in the context of the infi-net, that is because of the foundational internality which characterizes the fourth dimension. Here the culture is subsumed by what is inherently trans-cultural - something of such integrative power that it provides a common space for potentially all cultures. In other words, the fourth dimension - the inter- or trans-cultural - is internal in an ultimate sense; and as one may suspect, this super-transcendent category resonates with the disciplinary orientation of religion (to be discussed in due course). In any case, the self-society dimensional hierarchy does seem to work as a viable strand in the infi-net insofar as it can be correlated with the primary set of metacategories in the top row of the matrix.

\subsection{Fundamental Cultural Assumptions}

With respect to the next row - the one associated with Fundamental Cultural Assumptions - a full elaboration would require that correlations be established, not only with respect to the metacategories at the top of the infi-net, but also with respect to the Self-Society row just discussed. However, given that limitations of space preclude such a detailed discussion, our focus must be on links to the meta-categories.

Fundamental cultural assumptions, defined as the underlying presuppositions we bring to discourse, are not always things of which we are conscious, but they play a crucial role in the network of agreements and disagreements that define our approach to the world and our interactions with others. The assumptions achieve a high level of articulation in what are called classic texts - texts that are read and re-read precisely because of their ability to bring these assumptions to light even though interpreting such texts is often the occasion for heated debate. (By texts here I include not just those of a literary nature, but also works of music, architecture, painting, etc.) Moreover, while such assumptions can be personalized in a manner that makes them unique to an individual (i.e., Fundamental Personal Assumptions), the possibility of personalization does not make them any more conscious than the broader cultural variants. The point is that consciousness of our assumptions allows us to critique them and possibly to avoid or to rise above disputes based on ignorance of these assumptions - cultural or personal.

Given that assumptions are cultural in nature, they can radically differ from each other with respect to time and space. That is, they can change in the course of historical evolution, and they can vary significantly from one civilization to the next (e.g., Western Civilization, South Asian, East Asian, Meso-American, etc.). Indigenous (or non-urban) cultural forms should not be excluded from having their fundamental cultural assumptions brought into a comparative relationship each other or with their non-indigenous counterparts. But in order to facilitate such comparisons, the infi-net must develop categories whereby the assumptions can be organized; and that means relating them to the same meta-categories (i.e., Emergent Externality, Emergent Internaltiy, etc.) that served to structure the self-culture dimensional hierarchy.

Considering that birth is a kind of emergent phenomenon - a phenomenon where one emerges from an internal space (the womb) into an external world and that birth is the sine qua non of life - assumptions centering on that crucial reality will figure prominently in any cultural configuration. In other words, fundamental cultural assumptions about life will fit under the rubric of Emergent Exterrnality. To the extent that life is characterized, not only by birth, but also by an individual's growth, the emergence continues in the sense that growth sees the qualities of person rise out of their life experience. 
The externalist aspect of this growth will naturally pertain to assumptions regarding how life challenges are met, with the ultimate challenge being how one faces the externalist reality of death. How is death external? It is outside of us as it is our very negation, which reduces us to the fragments that are the mark of externality.

To give an example of how a classic text might illustrate emergent-externality assumptions, we can turn to Sophocles' famous play, Oedipus the King. The assumptions I will be putting forward are, of course, interpretations, but fundamental cultural assumptions can only be drawn from texts by hermeneutic discourse that in the context of the present discussion is informed by the infi-net. Thus, with regard to emergence, a pertinent assumption (and it is not the only one operative in the play) may be stated as: 'A person's emergence in terms of character development is a result of his/her life choices, where choices made on the basis of a prideful ignorance are likely to lead to a tragic outcome.' The protagonist's emergence here is a transformation from a somewhat arrogant ruler to one who is humbled by a terrible truth that he unknowingly killed his own father and married his mother - a truth which he is driven to pursue. (Sophocles, p. 114)

With regard to externality, a key assumption may be stated as: 'Life is in the grip of a powerful external force called fate; and while humans might know it to a degree, they cannot really alter it.' In other times the external forces controlling a person's life may be something like genetic endowment. Yet in all cultures and in all times there is the inevitable fate of death, which is external to the reality of life as its inescapable negation. Thus, with respect to emergent externality, fundamental cultural assumptions will tend to center on life as an emergent phenomenon and death as its external boundary.

Shifting from external to internal emergence, the fundamental cultural assumptions that best resonate with this meta-category are those associated with freedom-slavery. Here the classic texts talk about establishing a common space (or internalist realm) where the tensions between individuals have been resolved or are at least significantly muted. The weight of external necessity has also been lightened insofar as the possibilities of an easier life may 'emerge' from a system of human co-operation where class structure does not lead to significant degrees of oppression. Thus, if the common space established is a sphere of freedom, the very organization of this structure is never far away from the danger of slavery when the structure becomes one where freedom of the few is established at the expense of the many.

As an example of this freedom-slavery dynamic, one can turn to the classic text of Herodotus (The Histories) where Persian tyranny (under Xerxes) is contrasted with Greek freedom as articulated by the ex-king of Sparta. Addressing Xerxes directly, the Greek ex-king (Demaratus) explicitly expresses the nature of Greek freedom and implicitly contrasts it with Persian slavery: "They [the Greeks in general, but the Spartans in particular] are free - yes - but not entirely free; for they have a master, and that master is Law, which they fear more than your subjects fear you. Whatever this master commands, they do; and this command never varies: it is never to retreat in battle, however great the odds, but always to stand firm, and to conquer or die." (p. 141) Of course, not all Greeks are free despite this 'law'. Their society is one that allows slavery. Nonetheless, as law evolved to expand the range of a common space where some do not experience disempowerment with respect to others (e.g., women, the impoverished, outsiders, etc.), to that extent has the scope of freedom been increased. It is a process that goes on to this day, and in that sense freedom (as articulated in classic texts that discuss human rights, power, law, etc.) is an emergent and not a foundational phenomenon.

Foundations, then, come into play with two kinds of fundamental cultural assumptions: those associated with wisdom-folly, which are externalist in nature, and those associated with good-evil, which are more internalist. Wisdom, of course, is the main theme of the present discussion, and it is not without significance that it is situated within the infi-net along with its natural opposite, folly. How is wisdom foundational and external at the same time?

Turning to the almost archetypal example of Socrates (i.e., archetypal with respect to the problem of wisdom), we have a search for foundations mediated by dialogue or Socratic questioning: how does one really know what one is talking about? What is the foundation of one's claim that one knows anything? Folly is the occasion for one presuming that one's knowledge of a specific thing entitles that individual to speak authoritatively about other things, including the nature 
of reality (i.e., of foundations). Plato, Socrates' student and arguably the founder of the Western philosophical tradition, did have a theory of foundations, which are the mind-like and rational forms that underlie all things. But while these forms are internalist in their non-physical nature, they are decidedly externalist in their being outside normal human consciousness and, indeed, external to the physical realities they somehow underlie. Moreover, inasmuch as these forms are eternal, they cannot be thought of as emergent in any way. Wisdom, then, entails grasping the foundations of the world by adopting a position whereby one is external to or outside of it. Folly, on the other hand, is being deceived (i.e., fooled) by appearances, to which one is too close as is the case with prisoner's in Plato's cave (discussed in the Republic), who would have to go outside the cave to gain a sense of the foundations of reality. (Plato, pp. 345-348)

Now I do realize that utilizing the Socratic-Platonic exemplification of foundational externality may appear too convenient. In this regard, other wisdom-folly classics would need to be examined to vindicate the value of the meta-category being discussed. While I believe this can (and should) be done, my goal here is to give a basic presentation of the infi-net and illustrate how it has snared such big fish as Socrates and Plato even though I realize that there are many other big fish in the sea. If too many significant classic texts (expressing the fundamental cultural assumptions I am trying to co-ordinate) do not fit, the infi-net might indeed have to be re-woven. Wisdom here is giving integrative possibilities a chance. The wise-guy is often too quick to criticize and tear the infinet apart because it seems too contrived. I am not saying that every critic is to be denigrated as a 'wise-guy'; but it should be kept in mind that because criticizing is easier than creating, it can become a destructive temptation.

Moving then from foundational externality to foundational internality, fundamental cultural assumptions about good and evil rise to the fore. But what are evil and good? At a common sense level, if evil is to be associated with destruction (physical and/or psychological), then good may be connected to its opposite, which is creation or the power of creativity. The good-creativity link is well articulated in the classic text, Genesis, where the creative power, personified as God, functions as the foundational principle of the world and every facet of creation is deemed to be 'good'. (Genesis, 1,1-26) It is as if creation were an expression of positive valuation (i.e., goodness), and this principle (or assumption) suggests that we create what we value and value what we create. Contrariwise, we destroy what we devalue and devalue what, for whatever reason, we feel compelled to destroy. How destruction and creation are intertwined is a matter of no little subtlety and probably parallels the good-evil relationship. (Does destruction make way for acts of creation? Does the experience of evil allow us to clarify the nature of goodness?) But, as the creation-story of Genesis suggests, creation-goodness is clearly foundational with respect to the being of the world.

To complete the correlation of good-evil with the meta-category of Foundational Internality, we must now address the internality aspect, and again Genesis provides us with the appropriate fundamental cultural assumption - namely, that the culmination of 'good-creativity' is the common space of non-alienation called 'the garden of Eden'. (Genesis, 2, 4-18) At a more general level, the idea of an internalist foundation is not confined to the Old Testament. The assumption of a golden age being the foundation of humanity is well known, but its connection with internality is not. If one is in a common space with God (the creator) or nature (a creative force underlying all things), there can be none of the alienating externality that comes from being in some kind of disharmony with that foundation. A paradisical life may then best be understood as a kind of perfect common space characterized by the absence of any kind of alienation or separation from what gives life a sense of being blissful.

Before turning to the third row of the infi-net, that of disciplinary orientation, it is important to pause and consider how the correlation being put forward serves the cause of achieving a greater degree of wisdom in our lives. First of all, that wisdom-folly is itself only one of four sub-categories of fundamental cultural assumptions suggests that it is wise to contextual wisdom itself and not take it as some kind of absolute that stands above all other categories or forms of discourse. Secondly, awareness of a context that is neither arbitrary nor chaotic (i.e., the infi-net itself) allows us to rise above our place in the network by understanding where we and our 'opponents' are coming from and consider that these places are themselves inter-connected in subtle ways. Thirdly, if our world is disharmonious because of a rigid adherence to specific assumptions, the infi-net may be used as 
a tool to encourage a more flexible stance.

\subsection{Disciplinary Orientation}

Overly rigid adherence to fundamental cultural assumptions is not the only reason for disharmony or failure to develop a more progressive form of discourse. The fact that we approach the world from different disciplinary perspectives stands in the way of developing a more integrative perspective that the wise person seeks but the wise-guy rejects. Disciplines are ways of knowing, but I consider a wise-guy as someone who discounts some of these ways of knowing as illegitimate or inferior with respect to others. Wise-guys may be forgiven for being presumptuous in this regard, for their narrow historical perspective tends to blind them. Thus, while a religious 'discipline' might have been a pre-eminent way of knowing reality in the Middle Ages, the key disciplinary approach of Modernity is that of science. A more integrative approach is not just to harmonize what seem to be contradictory disciplinary orientations, but to explore how these could be interwoven with others (art and philosophy) in the context of the infi-net. The question is how could science, art, philosophy, and religion be correlated with the meta-categories and by way of that correlation also be linked with the categories of the first and second rows.

The connection between the discipline of science and emergent externality is fairly straightforward. With respect to externality, science has a physical or objective world as its primary object of knowing. That world is not known from 'within' (via the mind) but from 'without' in the sense that an experiment conducted in the external world must be the criterion of establishing truth. Theories couched in mathematical form are more internal than external, but even the most elegant and beautiful theory must pass the test of lining up with the realities of the external world as mediated by experiment.

At this point one might think that 'emergence' is, with respect to science, less relevant than the search for foundations, and that I am accordingly in error in placing science under the 'emergent externality' rubric. Yet is not most of science essentially emergent insofar as psychology may be said to emerge from biology, which emerges from chemistry, which emerges from physics? But even in physics, as the foundational science, emergence seems hard to avoid because of the externalist emphasis on cause and effect. If a cause is external to an effect, then even if atoms were foundational, a scientific understanding would be incomplete without knowing what the cause of these atoms might be. I am, of course, aware that atoms emerge out of a relationship between protons, neutrons, and electrons, and that these entities have their foundation in quarks. But inasmuch as it is far from clear that the latter are the ultimate foundations, emergence in an externalist vein seems to characterize the disciplinary approach to knowing called science.

Turning next to the to the disciplinary correlate of emergent internality, I place under that meta-category those ways of knowing the world associated with painting, sculpture, literature, architecture, music, film-making, etc. - that is to say, in the most general sense, art. While it might seem odd to think of art as a way of knowing the world, that might be because in the current cultural context the externalist sciences hold such a prominent position. However, from the perspective of the infi-net it would be foolish to deny that 'representing' the emergent world (via painting, prose, and poetry) is a way of 'knowing' it, and that such knowing is 'disciplined' albeit in a different way than that of the sciences.

To understand the 'difference' associated with internality and the 'similarity' associated with emergent realities, let us consider each of these in turn. But let us also remember, that like all strands of the infi-net, the movement from science to art is characterized by the quality of a continuum and not a disjunction. Thus, just as science aims at foundations without necessarily reaching them, it also has a certain orientation to what is thought more germane to the arts, that is, a sense of beauty with respect to how 'true' theories should display a quality of elegance.

What then are the internalist qualities associated with art understood in the most general sense?

First, despite its connection to science, the quality of beauty that characterizes most artistic expression has the following internalist associations: (a) To the extent that beauty entails the subtle integration of the elements constituting a given work of art, to that extent has the artist created a 
common space in the world and may even be suggesting that the world has many other integrative or common-space aspects. In other words, while the external world might have a latent common space quality, the artist makes it manifest in the work of art, where form and content speak to each other endlessly in a manner that downplays their separation and emphasizes their hidden unity that is, their internalist common space. (b) This sense of common space extends beyond the work and includes the viewer-listener-reader in a way that does not usually take place in the sciences. Not only does the audience enter into a common space with the alienation-reducing expression of the beautiful, the audience enters into a common space with each other who are bound together in appreciation and admiration of a work that might bestow a greater degree of meaning in their lives.

Secondly, apart from beauty as an internalist quality connected to the way an artist knows the world (or some aspect thereof), there is also the common space associated with the subjective engagement of the artist that contrasts strongly with the objective detachment a scientist must adopt with respect to knowing the world (or some aspect thereof). The latter stance cannot be tainted with common-space internality. The former stance requires the artist to balance objectivity with a closeness or intimacy that not only brings the artist into a common space with what is being explored, but also that extends to the audience, which is invited into that common space as well.

Thirdly, while imagination plays a significant role in the development of scientific knowing, it is limited by the rigors of reason and the need to keep theory in line with the realities of the external world. Art also must bend to the requirements of its media (sound, color, etc.), but the imagination is much more free to alter these to internalist possibilities associated with creativity and beauty. Inasmuch as the common space of consciousness is that locus of imaginative possibilities, artistic knowing again shows its internalist aspect.

Of course, the foregoing differences between the scientist and the artist must also be balanced by a powerful similarity that is associated with the meta-categorical theme of emergence that, according to the infi-net, applies to both of these ways of knowing. Just as science starts its project of knowing by working with such emergent phenomena as matter, motion, etc., art begins its project of knowing with such emergent phenomena as color, sound, texture, words, etc. Both ways of knowing can point to foundations, but even these can only emerge by way of (a) a theory that is never final, and (b) some kind of aesthetic theme, image or archetype that refers to the foundation without really making it present. Indeed, the conceit of foundations being actually present in some representation of God is tantamount to idolatry and thus forbidden in certain religious traditions (e.g., Judaism, Islam, and some Protestant sects of Christianity). Contrariwise, one could argue that Michelangelo's depiction of God, in the Sistine Chapel's famous ceiling fresco, allows the viewer to feel the immense distance between themselves and the foundation being represented by the artist. In any case, it is a fine and often tensive line in both ways of knowing. The famous adage in quantum physics, addressed to those who wish to flirt with foundations, is to 'calculate and shut-up'; but just as not all physicists adhere to this admonition, not all artists can refrain from attempting to go beyond emergent phenomena and bring (channel?) foundations into being. (Rosenblum and Kuttner, pp. 123, 141, 174-176)

When it comes to dealing more directly with foundations, there are two disciplinary possibilities: foundational externality and foundational internality. The former may be correlated with a philosophical way of knowing, the latter with a religious approach. Let us look at each of these in turn.

By 'philosophy' I do not just mean the discipline in and of itself. I would include the philosophy of history, of law, of science, etc. What all of these have in common would be a search for overviews by which various emergent phenomena are seen from a unifying perspective with a view to getting a sense of what might underlie them, what they have in common, what brought them into being - in short, what are the foundational realities at play. Within philosophy proper, a good example of a foundational reality would be Platonic forms. Another would be Lucreatian atoms and the void. (Lucretius, p. 458) Note that the emphasis here is not specifically on how these foundations manifest as emergent phenomena. It is on the foundations themselves and why they should be taken as such. The knower, in effect, is exploring the limits of the knowable and how that ultimate limit might inform various kinds of emergent phenomena.

What makes this disciplinary approach essentially external in comparison to that of religious 
knowing is its implicit or explicit claim to not being subjective. The philosophical exploration of foundations articulates positons, which others are invited to share because they have been convinced by the philosopher's arguments. While this goal of generating agreement might appear to be internalist, I do not believe that such is the philosopher's main intention. What is this intention? I believe it is the pursuit of an underlying truth that will allow the receivers of such truth not to bond more tightly together in an internalist community of 'believers', but to see as individuals some principle that is outside of (external to) themselves. The truth in question might be pleasant or unpleasant in itself and in its implications, but as truth it is essentially outside of us and in that sense it must always be sought. If it is deemed to have been absolutely found or framed in a manner that reduces it to some kind of rigid system (i.e., ideology), then the liberating potential of truth is in danger of becoming an enslaving lie. Real or authentic philosophy, then, is essentially endless, and acceptance of that is the core of its discipline - a discipline that is difficult to maintain.

Who does not want to possess the truth rather than endlessly seek it because it is essentially outside of us? Perhaps that is the core of Socratic wisdom - to endlessly question one's assumptions, to go outside of them no matter how challenging and painful that may be, to realize the 'truth' that one does not know the underlying foundational truth and that perhaps one can never know it, but to pursue it anyway. No wonder Socrates was perceived and, in a sense, was even portrayed as a kind of wise-guy. If, as an archetypal philosopher, Socrates' struggle with truth were taken too seriously, would not the rest of us feel ashamed in our indifference to (or fear of) the truth? Better he be the wise-guy than that we acknowledge our self-betrayal as would-be truth seekers.

If the search for an external truth characterizes foundational externality, then the realization of an internal trust or faith characterizes the way of knowing associated with religion. I use religion rather than theology, not simply because theology is a special branch of religious knowing, but also because, while theology might talk about faith or trust as a way of knowing, religion is the actual discipline of trust that has relevance to the infi-net. It might seem odd to even consider trust or faith as a way of knowing and having a disciplinary component, but it is the meta-categorical structure of the infi-net that calls on us to explore this possibility.

With respect to being foundational, religion clearly embodies that aspect of knowing. To know God as creator is to know the ultimate foundation, but by its very nature as a supreme, infinite, trans-human intelligence, God cannot be grasped by our finite intelligence. However, for some readers, the fact that there is even a place on the infi-net for such a form of knowing is enough to discredit my project outright. While I myself am not religious, I do not take my own limitations as some kind of absolute, and I would rather see what arises when one follows the structure of the infinet.

The key question, then, is how the foundational form of knowing associated with religion is connected to internality. Hints are provided by such expressions as 'the kingdom of God is within' and 'atman (the soul) is Brahman (the spiritual foundation of all things)'. From these two expressions alone, one can surmise that internal is somehow the route to a kind of foundational knowing.

But what does it mean to know the foundation(s) internally - that which underlies all emergent phenomena? To begin, it is a way of knowing that is radically different from its externalist variant, which utilizes human categories of understanding. The internalist mode of knowing 'infinitudinal' foundations is keenly aware of the limitations of these human or 'finite' categories, but it is ready to abandon them and thus approach or let oneself as knower be approached by the foundational reality. In other words, without the distancing or objectivizing categories of an externalist knowing of foundations, there is a possibility, if not necessity, of experiencing a common space with the source of all being. Secondly, one should not underestimate the fear-factor in knowing foundations in such an internalist manner. For giving up one's human categories or daring to put them aside because they are essentially inadequate is to give up a primary form of defense in approaching not only the sphere of emergent phenomena, but also something incomparably greater. Thus, it is fair to speak of knowing by engendering a sense of trust in the power of the foundation not to crush the 'conceptually naked' would-be knower.

In view of the claim I am thus making about the radical nature of religious knowing, I am 
bound to qualify the foregoing remarks by asserting that I am referring to an authentic religiosity and not the multitude of corrupt forms that my critics would, with some justification, take to be the norm. That is (as the critics might argue), most religious knowing is false in the sense that those who adhere to it are approaching the ultimate with well-worn categories which they are using as a shield to protect themselves. Hence, they are not really rendering themselves vulnerable with a view to experiencing a common space with foundations. More to the point, those criticizing the integrity of this infi-net category (i.e., Foundational Internality manifesting itself as religious knowing) would also assert that there is no evidence of internalist foundations in the first place and that everything can be explained at some point by externalist ones.

My response to such criticism must be here put forward lest the logic of the infi-net be called into question. First, with respect to a lack of evidence with respect to the foundational internal, I can only say that if the reality at question is essentially internal, then a demand for an external manifestation makes no sense; for by definition the internal (foundational or otherwise) cannot present itself in the external world or it would not be internal. Secondly, for those who have experienced the 'foundational internal' by giving up the 'human' categories that stood in the way of such apprehension, the inability to express or explain what they learned (or come to know) should not be taken as a sign (by the critics) that there is nothing to know. Again, if normal categories have to be abandoned or stretched to the limit, that is because the ones taken for granted by the critics are essentially inappropriate or inadequate. Thirdly and finally, in response to critics being skeptical of foundations that are overly anthropomorphized (e.g., the Abrahamic God) and hence an invitation to an infantile relationship to foundations, I answer that it is important not to judge such relationships from the outside, from the position that an externalist would take on the basis of not really having had an internal connection with foundational realities. If the religious knower experiences the relationship with the foundational Father as a call to responsibility, there is nothing infantile here.

Let me conclude this section by bringing together the two forms of foundational knowing external and internal -- in their disciplinary aspects. The key term here is that of 'enlightenment'. In the Western and external sense the discipline is that of subjecting what one would know to the strictures of a critical reason. It is a hard discipline since it involves dismantling the comfortable irrationalities that held society together, and Voltaire is probably the most prominent practitioner of this approach. In the Eastern and more internal sense the discipline of enlightenment is facilitated by such internalistic practices as meditation. It is also hard (if not harder than the Western form) since it involves dismantling not only the desires that underlie human suffering, but also the conceptual structures such as the 'self' that stand in the way of achieving a oneness with being. The Buddha is perhaps a counterpart to Voltaire in representing the Eastern enlightenment tradition. Voltaire did not develop the Newtonian science that led to mastery of the external world, but he did incorporate that outlook as part of a greater vision that allowed him and other philosophes to rise above or transcend their world in a critical and hence external sense. The Buddha, on the other hand, linked enlightenment, not to rising above the world, but to awakening to one's inherent unity with it - a form of knowing foundations that embodies authentic religiosity. Indeed, one might call Buddhism a purer form of religious knowing because it appears more directly aware of the snares of the 'self' concept (e.g., the 'soul' in the Abrahamic traditions) than are those religions which hold onto it and thereby weaken possibilities for total integration (i.e., 'selflessness') with the underlying foundations of being (Hagen, pp. 125,142).

\subsection{Primary Relation to Reality}

As for the last row of the infi-net (the one associated with 'Primary Relation to Reality'), it is, of course, not really the last. Any number of quaternal structures may be placed beneath the four primary meta-categories with a view to generating new integrative possibilities. However, the fourth row is a good summary for the present discussion because it touches on what may lie behind one's choice of discipline with respect to how one wants to know the world. One may think of the fourth row as a set of psychological categories that shed light on the way we choose to know the world, but also on the assumptions we choose to adopt or favor as well as those to which we are blind. If 
the wise person is one who knows her- or himself, understanding one's basic attitude to reality is of no small importance. More to the point, the infi-net illustrates how these fundamental attitudes may be interconnected and, in so doing, opens up the possibility of having more constructive conversations with those who have a different primary relation to the real.

Starting again with Emergent Externality, already correlated with the intra-personal, life-death, and science, we have as a psychological orientation to reality what I would call a propensity to mastery and control. This particular correlation is not hard to understand, given that emergent externalities confront us on a daily basis; and if not mastered or in some way controlled, we fear that they would overwhelm us. While the range of human mastery in general has increased with the development of scientific knowing, it also increases in the context of human growth from infancy to adulthood. For some individuals and no doubt for some cultures at certain times in their historical evolution, mastery remains a primary orientation, but that does not mean that other relationships to reality do not play significant roles. It is a question of using the infi-net to get one's bearings in this regard.

When it comes to emergent internalities of different communities or the internality associated with the pursuit of beauty, those focused on developing personal engagements with others or with some aspect of nature are oriented to intimacy as a primary orientation to reality. The pursuit of those common spaces that afford a sense of intimacy do come at a price - the price of feeling alienated from those who do not share that space. Likewise the pursuit of the intimacy associated with generating beauty in the world may come with an alienating revulsion from that which is deemed repulsive. The mastery orientation may work with intimacy-seeking one, but they are not the same; and a better understanding of how both of these operate may facilitate a certain wisdom in one's approach to the world. Thus, if attempts at intimacy fail, there could be a turn to an alienating mastery over others -- a possibility that might be minimized if one were guided by the infinet in one's choices.

Yet humans normally want more than the security afforded by mastery and intimacy. There is always the quest for meaning that arises out of a relationship to foundational realities, and we must now turn to the psychological orientation that comes into play here.

With respect to foundational externalities, there is a certain passion for vision, that is, a wish to see the whole by transcending one's narrow perspective albeit without giving up our human categories. In other words, this psychological orientation bespeaks a desire to transcend, to view the whole (foundations) from a distance (external). Mastery is not appropriate here because one cannot master the whole. Neither can one be intimate with or close to it. But one can endlessly struggle to increase one's range of vision and, in so doing, find meaning in the experience of ongoing transcendence.

Finally, we have to consider the psychological orientation to reality that may be correlated with a focus on foundational internalities. The personal internality of consciousness points the way. That internality is foundational for ourselves, but it is also divided and hence not perfectly unified. Perhaps the main division is that between sleep and wakefulness - something which suggests that an ongoing wakefulness not punctuated by episodes of sleep (lesser consciousness or nonconsciousness, yet still part of the internal sphere) represents a deeper layer of internality. Wakefulness, which may be associated with enlightenment in the Eastern sense, appears then to be an internalist path to the foundations that is achieved by perfecting the common space of internality - not by staying awake in some physical sense, but by removing divisions or fissures within the internal sphere. Because the final source of division may be associated with holding onto that aspect of the internal world known as the self, letting go or surrendering that occasion for a defensive separation from the world is the essence of this particular relation to foundations. Perhaps surrender is what lies behind faith, which may also be understood as an internalist relationship to a foundational reality personified as God.

From the perspective of the infi-net, these four primary relations to reality are not isolated from each other but are themselves part of what I would call a symmetrical expression of a more inclusive relationship to reality as well as to each other: mastery (emergent externality) is a symmetrical inversion of surrender (foundational internality), while intimacy (emergent internality) is a symmetrical inversion of transcendence (foundational externality). Symmetries associated with 
complementarity rather than inversion can be seen with respect to relationships between mastery and intimacy as well as between transcendence and surrender.

What has all this to do with the way of wisdom? If wisdom entails knowing oneself, the four categories associated with our primary relationship to reality offer a kind of mirror by which one might better have a better sense of one's relationship to reality at a fundamental level and by way of that get a more nuanced sense of who one is. Moreover, it also suggests how that relationship might be expanded as well as how one might be connected to those who seem to have a radically different connection to the real. In other words, I am suggesting that to know oneself is connected to knowing one's relationship to the world as well one's relationship to others. How? By utilizing the infi-net to get a sense of the whole of which we are all a part.

Yet there is more here than just a conceptual grid. It can hardly be effective if that grid does not inform educational practice. There is an organizational component at play here insofar as the infi-net serves as a tool for grasping the connections between fundamental cultural assumptions in different civilizations as these evolve over time. But there is also an experiential component wherein wisdom might be taught by exposing students, not only to different disciplinary orientations, but also to the differing psychological attitudes associated therewith by way of the grid. If I had to give this form of education a name, it would be not be inter-disciplinarity but integral-discipinarity. No doubt such an educational protocol would be very difficult. Yet, is that not to be expected if we wish to produce wise individuals as opposed to educated 'wise-guys'?

\section{What's the 'Catch'?}

It is time to review and assess what has been caught in the web of our net, and, as the heading suggests, ask if this is all some kind of trick. Is there a 'catch' to the infi-net, that is, a hidden cost to applying this web to the key problem of making ourselves wiser than we were before? I am obviously playing with two senses of the word 'catch'. On the one hand, we need to see what the web has caught in terms of ideas, concepts, meaning, etc. On the other hand, we need to consider whether there is a hidden cost ( $a$ 'catch') to this endeavor.

With respect to the first sense of the word, the infi-net has attempted to catch what normally is a source of division: assumptions, disciplines, primary relations to reality. But the infi-net has done this within the framework of a four-dimensional unity: the intra-personal, the inter-personal, the intra-cultural, and the inter- or trans-cultural; and in that sense the infi-net has caught these disparate elements in a unifying framework akin to the seamlessly unifying space-time framework that surrounds us in a physical sense. What the foregoing entails regarding the quest for wisdom is increasing the possibility of conversations that are not blocked or truncated within their respective contexts. Thus, the infi-net is meant to catch seekers and speakers - seekers of truth beyond their contextual confines, and those who are more inspired to speak their truth with a hope they will be better understood.

But here comes the second sense of 'catch', and it is connected to the educational protocols discussed at the end of the last section. The catch is that of time - the time to gain enough familiarity with the differing assumptions, disciplines, and approaches to reality to allow meaningful conversations to take place. At this point it is not clear what the degree of familiarity would have to be for an individual or group to understand the position of those speaking from a radically different context. However, because the point of the infi-net is precisely to facilitate such interchange by clarifying and integrating radically different contexts, the time for familiarization with the viewpoints of others might be reduced accordingly. It is simply too early to tell if this is a viable way of wisdom, but the achievement of a few breakthroughs in understanding based on infi-net principles might be a spur to motivation in this regard.

The second 'catch' is more subtle, and it is based on the limiting quality of the four metacategories. What if reality is best captured by another set - a set that may be five-fold or more? Is not any net distorting by way of its narrowness? While I would not oppose experiments with other kinds of net, one must be leery of letting the meta-categorical structure become too unwieldy. Moreover, its narrowness with respect to a primary set of meta-categories is balanced by the possibility of adding any number of rows (potentially an infinite number), whose elements would 
constitute a set of columns that would endlessly grow beneath each of the primary meta-categories. Thus, the catch of having a matrix that is overly narrow in one dimension is counterbalanced by having that same matrix potentially infinite in another.

\section{Conclusion}

With the foregoing provisos and admonitions, the infi-net may be cast upon the turbulent waters of human discourse. It may be vital to do so at a time when truth itself seems to be under siege. (See, for example, James Ball, Post-Truth - How Bullshit Conquered The World, London: Biteback Publishing, 2017.) In a world dominated by the Internet the spread of disinformation is all too easy, and in such a context media-savvy wise-guys will silence or edge out the voices of wisdom. Thus, in such extra-ordinary times we need some kind of method that will assist in the ongoing search for truth -- a method that goes beyond the exposing of lies and that seeks to explore the very foundations of disagreement. That exploration is the rationale of the infi-net.

I am not suggesting that all disagreement is wrong or futile. What is at issue with respect to wisdom is how we deal with such disagreements. In that sense the infi-net should be understood as a means to having good conversations, where a good conversation entails an increase in human consciousness and a bad one may be associated with a diminution of the same. My assumption here is that an increase in human consciousness is not just a way of dealing with the distortions and disempowerment that characterize a dysfunctional social order. A growth in consciousness may also be connected to the awakening of a sense of wonder - an awakening that motivates us to do the work of wisdom, which is in essence truth-seeking. How so? By the infi-net's integrative structure, one can explore and discover connections that one might never have thought possible and gain the realization that our differences need not be the occasion for a destructive silence but for a creative conversation.

\section{References}

Ball, James. 2017. Post-Truth - How Bullshit Conquered The World. London: Biteback Publishing.

Hagen, Steve. 2018. Buddhism Plain and Simple. North Clarendon, VT: Tuttle.

Herodotus. 1988. History. In Classics of Western Thought, Vol I - The Ancient World. $4^{\text {th }}$ Edition. Edited by Donald S. Gochberg, Boston: Wadsworth.

The Holy Bible - Revised Standard Version. 1973. New York: Collins.

Lucretius. 1988. On the Nature of Things. In Classics of Western Thought, Vol I - The Ancient World. $4^{\text {th }}$ Edition. Edited by Donald S. Gochberg, Boston: Wadsworth.

Plato. 1988. The Republic. In Classics of Western Thought, Vol I - The Ancient World. $4^{\text {th }}$ Edition. Edited by Donald S. Gochberg, Boston: Wadsworth.

Rosenblum, Bruce and Fred Kuttner. 2011. Quantum Enigma - Physics Encounters Consciousness. $2^{\text {nd }}$. Edition. Oxford: Oxford University Press.

Sophocles. 1965. Oedipus The King, Philoctetes, Electra, Antigone. Trans. Robert W. Corrigan.

New York: Dell Publishing Co. Inc. 